reviewed by Palliative Care team and compare the results with best practice guidance and the Gosport.

Methods The audit cohort was consisted of all patients who died in CCC between July 2018 and December 2018. 29 deaths were identified. Electronical medical records and paper prescription charts were reviewed regarding opiates and sedatives.

Results $84 \%$ of patients reviewed was not opioid na-ve on admission. $80 \%$ of patients were commenced on syringe driver during admission. Commencement of syringe driver was justified in $96 \%$. In the remaining 4\%, syringe driver use was appropriate, but it was not justified. Starting and finishing doses of opiates in syringe drivers were variable, whereas PRN opiate prescription was appropriately dosed in all cases. In 2 cases where the conversion was not according to the guidelines, rationale was given. Similar results were retrieved regarding midazolam prescription. Midazolam was prescribed in $90 \%$ cases in patients with syringe driver. Doses of midazolam in syringe driver ranged from $5 \mathrm{mg}$ to $60 \mathrm{mg}$ with appropriate justification of doses. There was no direct correlation between commencement of syringe driver and death. In $100 \%$ of cases, clear adherence to the clinical guidelines was shown.

Conclusion Current practice in prescribing opiates/sedatives and commencement of syringe driver is according to the clinical guidelines and dosing is appropriate. Results of this audit compared with results produced by the Gosport Independent Panel provide assurance about current use of these medications at CCC. Nevertheless, continuous training is necessary for prescribers in order to maintain the reassuring results.

\section{FINDINGS FROM THE FIRST ROUND OF THE NATIONAL AUDIT OF CARE AT THE END OF LIFE (NACEL)}

Suzanne Kite, Elizabeth Rees, Claire Holditch, Debbie Hibbert. NHS Benchmarking Network

10.1136/spcare-2020-PCC.90

NACEL is a national comparative audit of the quality and outcomes of care experienced by the dying person and those important to them during the last admission leading to death in acute, community hospitals and mental health inpatient providers in England and Wales. The audit, first undertaken during 2018/19, comprised:

- an Organisational Level Audit covering trusts (in England)/ Health Boards (in Wales)

- a Case Note Review completed by acute and community providers only, reviewing all deaths in April 2018 (acute providers) or deaths in April - June 2018 (community providers); and

- a Quality Survey completed online, or by telephone, by the bereaved person.

Data was collected between June and October 2018. 206 trusts in England and 8 Welsh organisations took part in at least one element of the audit (97\% of eligible organisations). A total of 11,034 case note reviews were included. Key findings included the following: -

- Documentation that a person may die imminently was high. For half of patients, imminent death was recognised less than one and a half days before they died, leaving a limited amount of time to discuss and implement an individual plan of care.
- People's experience of care was good, excellent or outstanding in most cases (80\%), as reported by the Quality Survey. However, $20 \%$ felt that there was scope to improve the quality of care and sensitive communication with both the patient and the family/others.

- Governance of end of life care was strong.

- Improvement is required in the documentation of an individual plan of care (documented evidence of a plan for $62 \%$ of people who died). Similarly, for one third of people who died, a discussion about the plan of care, and discussions about medication, hydration and nutrition had not been recorded.

The second round of NACEL is running in 2018/19.

\section{1 'IS THIS A CONVERSATION ABOUT DEATH?' PLANNING FOR FUTURE CARE WITH PATIENTS WITH ADVANCED CANCER: EXAMINING THE FEASIBILITY OF USING THE TRAJECTORY TOUCHPOINT TECHNIQUE FOR ADVANCE CARE PLANNING. A QUALITATIVE STUDY}

Daniel Lewin, Jessica Lee, Lynn Sudbury-Riley, Philippa Hunter-Jones, Stephen Mason, Farnaz Nickpour, Ahmed Al-Abdin, Sarika Hanchanale, Simon Roughneen, Amy Reed, Bob Giles, John Ellershaw. Royal Liverpool and Broadgreen University NHS Hospital Trust, University of Liverpool

\subsection{6/spcare-2020-PCC.91}

Background Advance Care Planning (ACP) enables people to make plans regarding their future healthcare and has been shown to positively impact on the quality of patient care. There are multiple barriers to engaging in the process of advance care planning. These include a lack of understanding of what ACP entails and patient, relative and healthcare professionals' anxieties in discussing potentially distressing issues. The Trajectory Touchpoint Technique (TTT) was applied to ACP discussions in order to create a visual aid to help circumvent these barriers, allowing deep and detailed discussions of potentially distressing topics.

Methods A literature review was performed to identify topics that patients may have wanted to talk about. These were ratified through a human centred design process (Design Thinking) with members of the public. ACP discussions were performed using the visual aid on an in-patient specialist palliative care unit. Qualitative enquiry was used in the form of patient, relative and clinician interviews following ACP discussions to evaluate the feasibility and benefits of using the visual aid.

Results Data was collected over a 4 week period in a single acute in-patient palliative care unit. Patients $(n=4)$, relatives $(n=2)$, and clinicians $(n=3)$ participated in the study. Thematic analysis of interview transcripts showed the visual aid enabled patients to begin the conversation by talking about the ACP related issues they felt comfortable with and that were most important to them. Relatives found the visual aid helped to identify what issues can be discussed. Clinicians found the visual aid helped focus the ACP discussions.

Conclusions We have demonstrated that the visual aid was useful in facilitating ACP discussions with this cohort of patients, relatives and clinicians. Further, we suggest these findings warrant trials across primary and secondary care for patients in end-of-life to further develop this approach to ACP facilitation. 